\title{
Índice de uniformidade de distribuição do amônio, nitrato, potássio e fósforo, em Latossolos sob condições de fertigação ${ }^{1}$
}

\author{
G uilherme K. Donagemma², Hugo A. Ruiz' ${ }^{3}$, Victor H. Alvarez V3, Paulo A. Ferreira ${ }^{3}$, \\ Reinaldo B. Cantarutti ${ }^{3}$, Agno T. Silva ${ }^{4} \&$ Getúlio C. Figueiredo ${ }^{5}$
}

\begin{abstract}
RESU MO
Se realizada de forma inadequada, a fertigação pode levar à salinização do solo ou lixiviação dos nutrientes, com a consequente contaminação do lençol freático. Neste sentido, se propõe, no presente estudo, um índice para determinar a uniformidade de distribuição de amônio, nitrato, potássio e fósforo, ao longo de colunas de Latossolos fertigadas, com o objetivo de nortear o manejo adequado da fertigação. Este índice foi calculado a partir de dados coletados em um ensaio de laboratório, seguindo-se um fatorial $4 \times(1+6)$, ou seja, quatro Latossolos de Minas Gerais (dois Latossolos Vermelho-Amarelos distróficos, um Latossolo Vermelho distroférrico e um Latossolo Vermelho distrófico), uma testemunha (aplicação de água deionizada) e seis formas de aplicação de $1 \mathrm{mmol}_{c}$ de N-N ${ }_{4}{ }^{+}, 1 \mathrm{mmol}_{c}$ de N-NO${ }_{3} ; 2 \mathrm{mmol}_{c}$ de K e $2 \mathrm{mmol}_{\mathrm{c}} \mathrm{dm}^{-3} \mathrm{de} \mathrm{P}$. Calcularam-se os coeficientes de variação para 0 teor de cada nutriente, ao longo das colunas e a relação entre 0 teor médio e o maior teor do nutriente (índice de uniformidade de distribuição de nutrientes) considerando-se 0 solo e 0 tratamento. Concluiu-se, portanto, que a relação maior teor, teor médio é um índice de uniformidade de distribuição de nutrientes adequado para avaliar a movimentação de íons com maior mobilidade no solo, como nitrato, amônio e potássio. 0 coeficiente de variação é menos adequado para mostrar a uniformidade de distribuição dos nutrientes.
\end{abstract}

Palavras-chave: dinâmica de íons, macronutrientes, nitrogênio, disponibilidade de nutrientes

\section{U niformity distribution index of ammonium, nitrate, potassium and phosphorus in fertigated Oxisols}

\begin{abstract}
Fertigation if conducted inadequately may cause soil salinization and consequently leaching of nutrients can pollute ground water. An index is proposed to determine the uniformity of distribution of ammonium, nitrate, potassium and phosphorus in columns of fertigated 0 xisols, which relates the highest content of the nutrient in a column segment with the mean nutrient content in the experimental unit. The higher the index value, the more irregular is the nutrient distribution. This index was calculated based on laboratory data evaluated in a $4 \times(1+6)$ factorial design including four 0 xisols of Minas Gerais (two distrophic Red-Yellow, one dystroferric Red and one dystrophic Red oxisol), one control (application of deionized water) and six application modes of application of $1 \mathrm{mmol}_{\mathrm{c}}$ of $\mathrm{N}-\mathrm{NH}_{4}{ }^{+}, 1 \mathrm{mmol}_{\mathrm{c}} \mathrm{N}-\mathrm{NO}_{3} ; 2 \mathrm{mmol}_{\mathrm{c}} \mathrm{K}$ and $2 \mathrm{mmol}_{\mathrm{c}} \mathrm{dm}^{-3} \mathrm{P}$. The coefficient of variation was calculated for the content of each nutrient along the columns. The ratio between the mean and the highest nutrient content was computed as well, considering the soil and the treatment. It was inferred that the ratio mean/highest content is an adequate index of uniformity of nutrient distribution to evaluate the movement of major mobile ions such as nitrate, ammonium and potassium in the soil. The coefficient of variation is less appropriate to show the uniformity of nutrient distribution.
\end{abstract}

Key words: ion dynamic, macronutrients, nitrogen, nutrient availability

1 Parte da Tese de Doutorado do primeiro autor, apresentada à Universidade Federal de Viçosa

2 Embrapa Solos, Rua Jardim Botânico 1024, CEP 22460-000, Rio de Janeiro, RJ. Fone: (21) 2179-4534. E-mail: donagemma@cnps.embrapa.br.

3 DPS e DEA/U FV. Campus universitário, CEP 36571-000, Viçosa, MG. Bolsista do CN Pq, Fone: (31) 3899-2630. E-mail: hruiz@ufv.br, vhav@ufv.br; cantarutti@ufv.br

4 INCAPER, Vila da Mata, CEP 29375-000, Venda Nova do Imigrante, ES, Fone: (28) 3546-1277. E-mail: agnosilva@yahoo.com.br

5 DPS/ESALQ, Av. Pádua dias, 11, CEP 13418-900, Piracicaba, SP. E-mail: getulioc@gmail.com 


\section{INTRODUÇÃO}

Para ser eficiente, a fertigação requer a localização dos nutrientes na profundidade de máxima densidade de raízes. Quando a lâmina de irrigação é insuficiente, os nutrientes podem se concentrar próximos à superfície do solo e, logicamente, as raízes crescem menos e exploram menor volume de solo, condições em que pode ocorrer o acúmulo de sais com consequente elevação da pressão osmótica da solução do solo acima de valores tolerados pelas plantas, diminuindo a produtividade, sobretudo em regiões semi-áridas e em cultivos em ambiente protegido. Por sua vez, a aplicação de uma lâmina de irrigação excessiva pode carrear os nutrientes para uma profundidade fora do alcance das raízes, diminuindo a eficiência da adubação; além disso pode, também, provocar a lixiviação desses nutrientes para o lençol freático, com consequente contaminação ambiental ou perdas econômicas.

Ressalta-se que o manejo adequado da fertigação compreende três etapas, em que a primeira se refere à aplicação de uma fração da lâmina de irrigação, a fim de permitir que a frente de molhamento atinja determinada profundidade; na segunda etapa se aplicam os fertilizantes dissolvidos na água de irrigação e a terceira etapa consiste em nova aplicação de água para lavar o sistema e transportar os nutrientes até a zona de máxima densidade de raízes (Frizzone et al., 1985; Carrijo et al., 1999; Pinto, 2001). Pinto (2001) sugere que se aplique um quarto da lâmina total de irrigação na primeira etapa, um meio na segunda e um quarto na terceira, porém, essas proporções não devem ser rigorosamente mantidas, pois o controle da profundidade e a localização dos nutrientes devem ser feitos por meio da quantidade de água aplicada na terceira etapa da fertigação (Hernandez, 1993). Com relação à movimentação dos nutrientes no solo, observa-se que há dependência de diversos fatores podendo-se citar, fundamentalmente, a concentração do nutriente em solução, relacionada com a capacidade de adsorção do elemento pelo solo (Grant \& Heaney, 1997; Qafoku et al., 2000), as cargas do complexo de troca iônica (Qafoku \& Sumner, 2001; Pinheiro, 2002), o pH (Qafoku et al., 2000), a solubilidade do fertilizante (Schumman, 2001) e as proporções dos nutrientes no fertilizante formulado (Silva, 2004). Por outro lado, a movimentação do nutriente depende do conteúdo de água (Fesch et al., 1998; Padilla et al., 1999) e da macroporosidade do solo (Kirkby et al., 1997; Jacobsen et al., 1997; Jensen et al., 1998; Shiptalo et al., 2000), dentre outros fatores. Na verdade, a movimentação de um nutriente no solo é resultado da atuação simultânea dos fatores supracitados e não da atuação isolada de um deles.

Percebe-se, então, que o movimento de nutrientes no solo é fundamental para se propor um manejo da fertigação que propicie a sua localização na profundidade de máxima densidade de raízes, aumentando, desta forma, a eficiência da fertigação e reduzindo a possibilidade de contaminação do lençol freático via lixiviação. Ressalta-se que a uniformidade de distribuição da fertigação, tanto na superfície quanto em profundidade, é interessante para o aumento da sua eficiência; neste sentido é oportuno estabelecer índices de uniformidade de distribuição de nutrientes ao longo de colunas de solo para nortear o manejo adequado da fertigação.
Objetivou-se, ante o exposto, estabelecer um índice de uniformidade de distribuição para os íons: amônio, nitrato, potássio e fósforo, em colunas preenchidas com Latossolos e fertigadas, para nortear um manejo perfeito da fertigação.

\section{MATERIAL E MÉTODOS}

\section{Delineamento e unidade experimental}

\section{Delineamento}

$\mathrm{O}$ delineamento experimental do presente trabalho foi em blocos casualisados, conforme um esquema fatorial $4 \times(1+6)$, sendo: quatro Latossolos de Minas Gerais: Latossolo Vermelho Amarelo distrófico textura média (LVAd1), Latossolo Vermelho Amarelo distrófico textura argilosa (LVA2), Latossolo Vermelho distroférrico textura argilosa (LVdf), Latossolo Vermelho distrófico textura muito argilosa (LVd) (Tabela 1); uma testemunha (aplicação de água deionizada) e seis formas de

Tabela 1. Caracterização física, química e mineralógica dos solos peneirados em malha de $4 \mathrm{~mm}$

\begin{tabular}{|c|c|c|c|c|}
\hline Características & LVAd1 & LVAd2 & LVdf & LVd \\
\hline \multicolumn{5}{|l|}{ Análise granulométrica $\left(\mathrm{kg} \mathrm{kg}^{-1}\right)$} \\
\hline - areia grossa ${ }^{1}$ & 0,25 & 0,27 & 0,21 & 0,06 \\
\hline - areia fina $^{1}$ & 0,53 & 0,14 & 0,09 & 0,15 \\
\hline - silte ${ }^{2}$ & 0,04 & 0,04 & 0,15 & 0,11 \\
\hline - argila $^{2}$ & 0,18 & 0,55 & 0,55 & 0,68 \\
\hline \multicolumn{5}{|l|}{ Densidade $\left(\mathrm{kg} \mathrm{dm}^{-3}\right)$} \\
\hline - do solo 3 & 1,24 & 1,03 & 0,96 & 0,88 \\
\hline - de partículas ${ }^{4}$ & 2,68 & 2,68 & 2,78 & 2,57 \\
\hline \multicolumn{5}{|l|}{ Porosidade $\left(\mathrm{dm}^{3} \mathrm{dm}^{-3}\right)$} \\
\hline- total $\left.\right|^{5}$ & 0,54 & 0,62 & 0,65 & 0,66 \\
\hline - macroporosidade 6 & 0,37 & 0,30 & 0,32 & 0,26 \\
\hline - microporosidade ${ }^{7}$ & 0,17 & 0,32 & 0,33 & 0,40 \\
\hline $\mathrm{N}-\mathrm{NH}_{4}{ }^{+}(\mathrm{mg} \mathrm{dm}-3)^{8}$ & 4,18 & 5,31 & 5,65 & 5,23 \\
\hline $\mathrm{N}-\mathrm{NO}_{3}^{-}\left(\mathrm{mg} \mathrm{dm}^{-3}\right)^{9}$ & 5,11 & 6,68 & 18,35 & 12,26 \\
\hline $\mathrm{K}\left(\mathrm{mg} \mathrm{dm}^{-3}\right)^{10}$ & 11,40 & 41,00 & 40,00 & 67,00 \\
\hline$P\left(m g m^{-3}\right)^{11}$ & 1,10 & 2,40 & 1,70 & 4,40 \\
\hline $\mathrm{Ca}^{2+}\left(\mathrm{cmol}_{\mathrm{c}} \mathrm{dm}^{-3}\right)^{12}$ & 0,01 & 0,03 & 0,39 & 0,56 \\
\hline $\mathrm{Mg}^{2+}\left(\mathrm{cmol}_{\mathrm{c}} \mathrm{dm}^{-3}\right)^{12}$ & 0,00 & 0,06 & 0,35 & 0,27 \\
\hline $\mathrm{Al}^{3+}\left(\mathrm{cmol}_{\mathrm{c}} \mathrm{dm}^{-3}\right)^{12}$ & 0,17 & 2,20 & 1,20 & 1,00 \\
\hline Carbono orgânico (dag $\left.\mathrm{kg}^{-1}\right)^{13}$ & 2,17 & 2,44 & 3,57 & 4,36 \\
\hline pH em água $(1: 2,5)$ & 4,95 & 4,97 & 4,44 & 4,80 \\
\hline pH em KCl $1 \mathrm{~mol} \mathrm{~L}^{-1}(1: 2,5)$ & 3,97 & 4,23 & 4,14 & 3,98 \\
\hline$\Delta \mathrm{pH}$ & $-0,98$ & $-0,74$ & $-0,30$ & $-0,82$ \\
\hline$P$ remanescente $\left(\mathrm{mg} \mathrm{L}^{-1}\right)^{14}$ & 49,49 & 8,29 & 6,34 & 12,17 \\
\hline \multirow{3}{*}{$\begin{array}{l}\text { Mineralogia da fração argila }{ }^{15} \\
\text { (Principais minerais, em proporção } \\
\text { decrescente) }\end{array}$} & $\mathrm{Ka}$ & $\mathrm{Ka}$ & $\mathrm{Ka}$ & $\mathrm{Ka}$ \\
\hline & $\mathrm{Gb}$ & $\mathrm{Gb}$ & $\mathrm{Gb}$ & Gt \\
\hline & $\|$ & VHE & $\mathrm{Hm}$ & $\mathrm{Hm}$ \\
\hline
\end{tabular}

${ }_{1}$ Por peneiramento (Ruiz, 2005); ${ }^{2}$ Método da pipeta (Ruiz, 2005); ${ }^{3}$ Método da proveta (EMBRAPA 1997); ${ }^{4}$ Método do balão volumétrico (EMBRAPA, 1997); ${ }^{5}$ Porosidade total $=1$ - (densidade do solo/densidade de partículas); ${ }^{6}$ Água retida entre 0 e $-6 \mathrm{kPa}$. (Ruiz, 2004); ${ }^{7}$ Água retida a $-6 \mathrm{kPa}$ (Ruiz, 2004); ${ }^{8}$ Dosagem com solução de salicilato de sódio (Kempers \& Zewers, 1986); ${ }^{9}$ Dosagem com solução de salicilato de sódio (Yang et al., 1998); ${ }^{10}$ Extrator Mehlich-1 (Defelipo \& Ribeiro, 1997); ${ }^{11}$ Extrator Mehlich-1 (Braga \& Defelipo, 1974); ${ }^{12}$ Extrator KCI 1 mol L-1 (EMBRAPA, 1997); ${ }^{13}$ Oxidação com dicromato de potássio e titulação com sulfato ferroso amoniacal (Yeomans \& Bremner, 1988); ${ }^{14}$ Alvarez V. etal. (2000); ${ }^{15} \mathrm{~Gb}$ : gibbsita, Gt: goethita, Hm: hematita, Ka: caulinita, Il: ilita, VHE: vermiculita com hidróxido-entre camadas. Referências para caracterização mineralógica: Amaral (2000) (LVAd2); Donagemma (2000) (LVdf); Santos Neto (2003) (LVAd1) e Araújo (1997) (LVd) 
aplicação do pulso ou pulsos de nutrientes (Kirkham \& Powers, 1972), sendo três repetições.

\section{Unidade experimental}

Conduziu-se o ensaio em laboratório utilizando-se colunas de percolação (unidade experimental). Cada unidade experimental foi constituída por 11 anéis de PVC com $6,6 \mathrm{~cm}$ de diâmetro interno e $7 \mathrm{~cm}$ de altura cada um, totalizando altura de $77 \mathrm{~cm}$. Os anéis foram sobrepostos e vedados com cola de silicone nas uniões e externamente, foram fixados com fita adesiva. A coluna foi preenchida com solo peneirado em malha de $4 \mathrm{~mm}$ e secado ao ar, até $7 \mathrm{~cm}$ da borda superior, formando uma coluna de $70 \mathrm{~cm}$ de altura e volume de $2,4 \mathrm{dm}^{3}$. Definiu-se a lâmina de irrigação após testes prévios, como aquela em que a frente de molhamento atingisse o nono anel da coluna de solo $(63 \mathrm{~cm}$ de profundidade), que é uma profundidade bem maior que os $20 \mathrm{~cm}$, e na qual, se concentram, em geral, as raízes que absorvem nutrientes.

\section{Aplicação das soluções}

A lâmina de irrigação foi dividida em cinco frações iguais $\left(\mathrm{F}_{\mathrm{i}}\right)$ e a dose dos nutrientes aplicada integralmente (D), ou fracionada em duas $\left(D_{1 / 2}\right)$ ou, ainda em três vezes $\left(\mathrm{D}_{1 / 3}\right)$, segundo o esquema: $\mathrm{F}_{2} \mathrm{D}, \mathrm{F}_{3} \mathrm{D}, \mathrm{F}_{4} \mathrm{D}, \mathrm{F}_{2} \mathrm{D}_{1 / 2} \mathrm{~F}_{3} \mathrm{D}_{1 / 2}$, $\mathrm{F}_{3} \mathrm{D}_{1 / 2} \mathrm{~F}_{4} \mathrm{D}_{1 / 2}$, e $\mathrm{F}_{2} \mathrm{D}_{1 / 3} \mathrm{~F}_{3} \mathrm{D}_{1 / 3} \mathrm{~F}_{4} \mathrm{D}_{1 / 3}$. A dose correspondeu a $2 \mathrm{mmol}_{\mathrm{c}} \mathrm{dm}^{-3}$ de $\mathrm{N}^{-} \mathrm{NO}_{3}{ }^{-}$e N-NH${ }_{4}{ }^{+}, \mathrm{K}$ e $\mathrm{P}$, com o emprego de $\mathrm{NH}_{4} \mathrm{NO}_{3}, \mathrm{KCl}$ e $\mathrm{KH}_{2} \mathrm{PO}_{4}$ como fontes, respectivamente (Tabela 2)

Tabela 2. Q uantidade de reagente aplicada nas colunas de $2,4 \mathrm{dm}^{3}$ de solo e quantidade de nitrogênio, potássio e fósforo resultante da aplicação de $2 \mathrm{mmol}_{\mathrm{c}} \mathrm{dm}^{-3}$ do elemento

\begin{tabular}{|c|c|c|c|c|c|c|}
\hline \multirow{4}{*}{ Reagente } & \multirow{3}{*}{$\begin{array}{c}\text { Quantidade } \\
\text { de Reagente }\end{array}$} & \multicolumn{5}{|c|}{ Dose } \\
\hline & & \multicolumn{3}{|c|}{ Nitrogênio } & \multirow{2}{*}{$\mathbf{K}^{+}$} & \multirow{2}{*}{$\mathrm{P}-\mathrm{H}_{2} \mathrm{PO}_{4}^{-}$} \\
\hline & & $\mathrm{N}-\mathrm{NH}_{4}^{+}$ & $\mathrm{N}-\mathrm{NO}_{3}^{-}$ & Total & & \\
\hline & \multicolumn{6}{|c|}{ mg por coluna } \\
\hline $\mathrm{NH}_{4} \mathrm{NO}_{3}$ & 192 & 33,6 & 33,6 & 67,1 & - & - \\
\hline $\mathrm{KCl}$ & 237,6 & - & - & - & 124,5 & \\
\hline $\mathrm{KH}_{2} \mathrm{PO}_{4}$ & 218,4 & - & - & - & 62,7 & 49,7 \\
\hline Total mg por coluna & - & 33,6 & 33,6 & 67,1 & 187,3 & 49,7 \\
\hline Total mmol dm-3 & - & 1,0 & 1,0 & 2,0 & 2,0 & 0,6 \\
\hline
\end{tabular}

\section{Desmontagem das colunas e análises químicas}

Após dois dias da aplicação da lâmina de irrigação, que era o tempo em que se garantia que a frente de molhamento chegava ao nono anel, as colunas foram desmontadas. Imediatamente, de cada um dos nove anéis superiores se retirou solo contido nos $5 \mathrm{~cm}$ centrais, utilizando-se uma faca, descartando-se o solo restante. Subamostras foram utilizadas para determinação colorimétrica do teor de N-NH4+ (Kempers \& Zwers, 1986) e do teor de N-NO3- (Yang et al., 1998), após extração com solução de $\mathrm{KCl} 1$ mol.L-1, e de K e P, extraídos por Mehlich-1, dosados por fotometria de chama (Defelipo \& Ribeiro, 1997) e por colorimetria (Braga \& Defelipo, 1974), respectivamente.

Essas doses foram adotadas de forma a fixar e de tal for- ma a serem concentrações que não fugissem a valores das recomendações de adubação feitas a campo.

\section{Cálculo dos índices de uniformidade e representação gráfica}

Considerando-se os teores dos nutrientes determinados em amostras de cada anel representou-se, graficamente, a distribuição nas colunas, relacionando-se esses valores com a profundidade média dos anéis $(3,5$ até $59,5 \mathrm{~cm})$, e se calculou o coeficiente de variação (CV) do teor dos nutrientes ao longo das colunas; posteriormente se estabeleceu, para cada repetição dos 28 tratamentos, a profundidade média do maior teor e seu valor numérico.

O índice de uniformidade (IU) de distribuição proposto foi calculado pela relação entre o teor médio e o maior teor dos nutrientes, com posterior análise estatística.

\section{Análises estatísticas}

$\mathrm{Na}$ análise de variância os graus de liberdade para tratamentos foram desdobrados em contrastes ortogonais, calculando-se os valores médios dos contrastes $(\overline{\mathrm{C}})$ (Alvarez V. \& Alvarez, 2006), pela Eq 1.

$$
\overline{\mathrm{C}}=\frac{\sum \mathrm{c}_{\mathrm{i}} \overline{\mathrm{y}}_{\mathrm{i}}}{\sum\left|\mathrm{c}_{\mathrm{i}}\right| / 2}
$$

em que:

$$
\begin{aligned}
& \mathrm{c}_{\mathrm{i}}-\text { coeficiente } \\
& \overline{\mathrm{y}}_{\mathrm{i}}-\mathrm{a} \text { média das determinações }
\end{aligned}
$$

\section{RESULTADOS E DISCUSSÃO}

Os valores dos coeficientes de variação apresentados pela Tabela 3, permitem evidenciar que, de maneira geral, houve um aumento a medida em que ocorria uma diminuição da mobilidade do nutriente na coluna; desta forma, o fósforo, que praticamente não sofreu deslocamento mas concentrou a dose aplicada no anel superficial, apresentou os maiores coeficientes de variação, praticamente sem alteração em resposta aos tratamentos impostos.

No caso dos nutrientes mais móveis, o maior deslocamento em profundidade com a concentração do pulso e a aplicação em frações iniciais de lâmina levaram a mostrar, em geral, menores valores do coeficiente de variação. Como se pode observar para o LVAd1, uma vez que neste solo predomina areia fina; dessa forma menor é proporção de carga negativa para reter o amônio comparado com um solo argiloso e com predomínio de carga negativa. Assim, o amônio se movimentou mais neste solo. Por outro lado, o LVAd2, por ser um solo argiloso, caulinítico, onde predominam cargas negativas, houve maior retenção do amônio, e assim o seu deslocamento foi menor e, consequentemente, maior valor do coeficiente.

No intuito de se comparar estatisticamente a variação na distribuição de nutrientes ao longo das colunas formulou-se, para os mesmos tratamentos, um índice que relaciona o maior teor com o teor médio do nutriente para cada unidade experimental. 
Tabela 3. Coeficiente de variação dos teores de amônio, nitrato, potássio e fósforo nos anéis da coluna, aplicados como pulso único, considerandose 0 solo e a fração da lâmina de irrigação (F) em que os nutrientes foram aplicados em dose integral (D), ou fracionados em duas $\left(D_{1 / 2}\right)$ ou em três vezes $\left(D_{1 / 3}\right)$

\begin{tabular}{|c|c|c|c|c|c|}
\hline \multirow{2}{*}{ Solo } & \multirow{2}{*}{ Tratamento } & $\mathrm{N}-\mathrm{NH}_{4}^{+}$ & $\mathrm{N}-\mathrm{NO}_{3}^{-}$ & $\mathbf{K}$ & $\mathbf{P}$ \\
\hline & & \multicolumn{4}{|c|}{ Coeficiente de Variação (\%) } \\
\hline \multirow[t]{7}{*}{ LVAd1 } & $F_{2} D$ & 12,86 & 44,82 & 51,86 & 184,10 \\
\hline & $\mathrm{F}_{3} \mathrm{D}$ & 16,74 & 37,29 & 72,78 & 185,62 \\
\hline & $\mathrm{F}_{4} \mathrm{D}$ & 18,89 & 42,16 & 85,77 & 183,52 \\
\hline & $\mathrm{F}_{2} \mathrm{D}_{1 / 2} \mathrm{~F}_{3} \mathrm{D}_{1 / 2}$ & 26,70 & 46,00 & 92,03 & 186,42 \\
\hline & $\mathrm{F}_{3} \mathrm{D}_{1 / 2} \mathrm{~F}_{4} \mathrm{D}_{1 / 2}$ & 27,70 & 48,16 & 103,11 & 186,11 \\
\hline & $\mathrm{F}_{2} \mathrm{D}_{1 / 3} \mathrm{~F}_{3} \mathrm{D}_{1 / 3} \mathrm{~F}_{4} \mathrm{D}_{1 / 3}$ & 31,36 & 63,40 & 111,33 & 185,81 \\
\hline & Média & 22,38 & 46,97 & 86,15 & 185,26 \\
\hline \multirow[t]{7}{*}{ LVAd2 } & $F_{2} D$ & 18,92 & 43,05 & 37,36 & 238,00 \\
\hline & $\mathrm{F}_{3} \mathrm{D}$ & 18,78 & 52,05 & 44,25 & 238,58 \\
\hline & $\mathrm{F}_{4} \mathrm{D}$ & 25,58 & 46,49 & 53,25 & 240,00 \\
\hline & $\mathrm{F}_{2} \mathrm{D}_{1 / 2} \mathrm{~F}_{3} \mathrm{D}_{1 / 2}$ & 29,96 & 47,54 & 56,95 & 237,96 \\
\hline & $\mathrm{F}_{3} \mathrm{D}_{1 / 2} \mathrm{~F}_{4} \mathrm{D}_{1 / 2}$ & 30,92 & 50,16 & 64,03 & 237,97 \\
\hline & $\mathrm{F}_{2} \mathrm{D}_{1 / 3} \mathrm{~F}_{3} \mathrm{D}_{1 / 3} \mathrm{~F}_{4} \mathrm{D}_{1 / 3}$ & 36,03 & 53,05 & 70,78 & 238,11 \\
\hline & Média & 26,70 & 48,72 & 54,44 & 238,44 \\
\hline \multirow[t]{7}{*}{ LVdf } & $F_{2} D$ & 22,51 & 31,32 & 42,42 & 162,40 \\
\hline & $\mathrm{F}_{3} \mathrm{D}$ & 23,00 & 30,90 & 47,89 & 164,35 \\
\hline & $\mathrm{F}_{4} \mathrm{D}$ & 26,12 & 40,42 & 55,01 & 165,52 \\
\hline & $\mathrm{F}_{2} \mathrm{D}_{1 / 2} \mathrm{~F}_{3} \mathrm{D}_{1 / 2}$ & 28,61 & 36,49 & 60,29 & 162,60 \\
\hline & $\mathrm{F}_{3} \mathrm{D}_{1 / 2} \mathrm{~F}_{4} \mathrm{D}_{1 / 2}$ & 32,21 & 42,44 & 65,14 & 162,03 \\
\hline & $\mathrm{F}_{2} \mathrm{D}_{1 / 3} \mathrm{~F}_{3} \mathrm{D}_{1 / 3} \mathrm{~F}_{4} \mathrm{D}_{1 / 3}$ & 35,29 & 45,23 & 66,04 & 164,91 \\
\hline & Média & 27,96 & 37,80 & 56,13 & 163,64 \\
\hline \multirow[t]{7}{*}{ LVd } & $F_{2} D$ & 28,10 & 43,26 & 27,47 & 117,50 \\
\hline & $\mathrm{F}_{3} \mathrm{D}$ & 26,15 & 38,89 & 30,13 & 118,20 \\
\hline & $\mathrm{F}_{4} \mathrm{D}$ & 28,55 & 35,01 & 33,35 & 118,38 \\
\hline & $\mathrm{F}_{2} \mathrm{D}_{1 / 2} \mathrm{~F}_{3} \mathrm{D}_{1 / 2}$ & 34,93 & 37,58 & 37,24 & 117,98 \\
\hline & $\mathrm{F}_{3} \mathrm{D}_{1 / 2} \mathrm{~F}_{4} \mathrm{D}_{1 / 2}$ & 35,74 & 48,82 & 42,18 & 120,12 \\
\hline & $\mathrm{F}_{2} \mathrm{D}_{1 / 3} \mathrm{~F}_{3} \mathrm{D}_{1 / 3} \mathrm{~F}_{4} \mathrm{D}_{1 / 3}$ & 38,90 & 53,14 & 45,44 & 121,40 \\
\hline & Média & 32,06 & 42,78 & 35,97 & 118,93 \\
\hline
\end{tabular}

Quanto maior o valor do índice maior também a desuniformidade na distribuição dos nutrientes na coluna; os resultados são apresentados na Tabela 4 e as comparações entre solos e tratamentos, dentro de cada solo, nas Tabelas 5 e 6 , respectivamente.

Observando-se as relações calculadas para o amônio, verifica-se que o índice apresenta valores na sequência: LVAd1 $<$ LVAd2 < LVdf < LVd (Tabela 4); Nesse sentido, para sua interpretação, devemos considerar à disponibilidade inicial deste íon nesses solos, que apresentou valores semelhantes (Tabela 1), assim, o valor crescente do índice para o amônio, do LVAd1 ao LVd, pode ser atribuído à textura desses solos, com proporção de argila aumentada nessa ordem; em referência à maior proporção de argila, maior também a interação dos cátions com o complexo de troca. Este resultados estão em acordo com Oliveira et al. (2004), que tambem observaram maior retardamento ao movimetno do amônio, quanto maior a proporção de argila no solo.

Com base no nitrato e na interação desse ânion com os sólidos do solo, esperar-se-iam índices na sequência LVAd1 $<$ LVAd2 < LVd < LVdf, sendo este último o material mais oxídico; contudo, a ordem foi LVdf $<$ LVAd1 $<$ LVd $<$ LVAd2
Tabela 4. Relação entre o maior teor e 0 teor médio de amônio, nitrato, potássio e fósforo nos anéis da coluna, aplicados como pulso único, considerando-se o solo e a fração da lâmina de irrigação (F) em que os nutrientes foram aplicados em dose integral (D), ou fracionados em duas $\left(D_{1 / 2}\right)$ ou em três vezes $\left(D_{1 / 3}\right)$

\begin{tabular}{|c|c|c|c|c|c|}
\hline \multirow{2}{*}{ Solo } & \multirow{2}{*}{ Tratamento } & $\mathrm{N}-\mathrm{NH}_{4}{ }^{+}$ & $\mathrm{N}-\mathrm{NO}_{3}^{-}$ & $\mathbf{K}$ & $\mathbf{P}$ \\
\hline & & \multicolumn{4}{|c|}{$\%$} \\
\hline \multirow[t]{7}{*}{ LVAd1 } & $F_{2} D$ & 1,23 & 1,61 & 1,87 & 7,35 \\
\hline & $\mathrm{F}_{3} \mathrm{D}$ & 1,27 & 1,56 & 2,66 & 7,36 \\
\hline & $\mathrm{F}_{4} \mathrm{D}$ & 1,33 & 1,63 & 2,98 & 7,40 \\
\hline & $\mathrm{F}_{2} \mathrm{D}_{1 / 2} \mathrm{~F}_{3} \mathrm{D}_{1 / 2}$ & 1,35 & 1,78 & 3,15 & 7,35 \\
\hline & $\mathrm{F}_{3} \mathrm{D}_{1 / 2} \mathrm{~F}_{4} \mathrm{D}_{1 / 2}$ & 1,40 & 1,91 & 3,45 & 7,35 \\
\hline & $\mathrm{F}_{2} \mathrm{D}_{1 / 3} \mathrm{~F}_{3} \mathrm{D}_{1 / 3} \mathrm{~F}_{4} \mathrm{D}_{1 / 3}$ & 1,50 & 2,07 & 3,65 & 7,35 \\
\hline & Média & 1,35 & 1,76 & 2,96 & 7,36 \\
\hline \multirow[t]{7}{*}{ LVAd2 } & $F_{2} D$ & 1,27 & 1,73 & 1,78 & 5,91 \\
\hline & $\mathrm{F}_{3} \mathrm{D}$ & 1,23 & 1,90 & 2,06 & 5,95 \\
\hline & $\mathrm{F}_{4} \mathrm{D}$ & 1,38 & 1,89 & 2,28 & 5,89 \\
\hline & $\mathrm{F}_{2} \mathrm{D}_{1 / 2} \mathrm{~F}_{3} \mathrm{D}_{1 / 2}$ & 1,44 & 1,93 & 2,38 & 5,97 \\
\hline & $\mathrm{F}_{3} \mathrm{D}_{1 / 2} \mathrm{~F}_{4} \mathrm{D}_{1 / 2}$ & 1,47 & 2,00 & 2,63 & 5,96 \\
\hline & $\mathrm{F}_{2} \mathrm{D}_{1 / 3} \mathrm{~F}_{3} \mathrm{D}_{1 / 3} \mathrm{~F}_{4} \mathrm{D}_{1 / 3}$ & 1,53 & 2,02 & 2,86 & 6,03 \\
\hline & Média & 1,39 & 1,91 & 2,33 & 5,95 \\
\hline \multirow[t]{7}{*}{ LVdf } & $\mathrm{F}_{2} \mathrm{D}$ & 1,32 & 1,55 & 1,84 & 5,33 \\
\hline & $\mathrm{F}_{3} \mathrm{D}$ & 1,37 & 1,50 & 2,15 & 5,44 \\
\hline & $\mathrm{F}_{4} \mathrm{D}$ & 1,39 & 1,61 & 2,39 & 5,41 \\
\hline & $\mathrm{F}_{2} \mathrm{D}_{1 / 2} \mathrm{~F}_{3} \mathrm{D}_{1 / 2}$ & 1,41 & 1,51 & 2,55 & 5,34 \\
\hline & $\mathrm{F}_{3} \mathrm{D}_{1 / 2} \mathrm{~F}_{4} \mathrm{D}_{1 / 2}$ & 1,45 & 1,73 & 2,65 & 5,32 \\
\hline & $\mathrm{F}_{2} \mathrm{D}_{1 / 3} \mathrm{~F}_{3} \mathrm{D}_{1 / 3} \mathrm{~F}_{4} \mathrm{D}_{1 / 3}$ & 1,60 & 1,80 & 2,68 & 5,40 \\
\hline & Média & 1,42 & 1,62 & 2,38 & 5,38 \\
\hline \multirow[t]{7}{*}{ LVd } & $\mathrm{F}_{2} \mathrm{D}$ & 1,46 & 1,77 & 1,65 & 4,13 \\
\hline & $\mathrm{F}_{3} \mathrm{D}$ & 1,40 & 1,76 & 1,73 & 4,15 \\
\hline & $\mathrm{F}_{4} \mathrm{D}$ & 1,42 & 1,62 & 1,81 & 4,16 \\
\hline & $\mathrm{F}_{2} \mathrm{D}_{1 / 2} \mathrm{~F}_{3} \mathrm{D}_{1 / 2}$ & 1,47 & 1,74 & 1,91 & 4,15 \\
\hline & $\mathrm{F}_{3} \mathrm{D}_{1 / 2} \mathrm{~F}_{4} \mathrm{D}_{1 / 2}$ & 1,52 & 2,04 & 2,05 & 4,20 \\
\hline & $\mathrm{F}_{2} \mathrm{D}_{1 / 3} \mathrm{~F}_{3} \mathrm{D}_{1 / 3} \mathrm{~F}_{4} \mathrm{D}_{1 / 3}$ & 1,64 & 2,11 & 2,15 & 4,24 \\
\hline & Média & 1,49 & 1,84 & 1,88 & 4,17 \\
\hline
\end{tabular}

(Tabela 4); esta aparente contradição pode ser atribuída à diferente disponibilidade inicial do nitrato (Tabela 1). A relação, para os quatro solos, na caracterização, foi de 3,59:2,40:1,31:1, na sequência LVdf:LVd:LVAd2:LVAd1; assim, o maior teor inicial de nitrato no LVdf e LVd contribuiu para incrementar o denominador do índice e, consequentemente, diminuir seu valor numérico. Essas considerações justificam os sinais dos contrastes (Tabela 5). Essa maior movimentação de nitrato quanto maior a sua concetração no solo, tambem foi observada em Latossolo Vermelho Amarelo (Costa et al. 1999).

Todas as comparações foram significativas, em que o LVAd1 apresentou a maior relação (Tabela 5).

Comparando-se a disponibilidade inicial, nota-se que as relações dos quatro solos foram de 5,98:3,60:3,51:1, para LVd, LVAd2, LVdf e LVAd1, respectivamente; então, esta disponibilidade inicial condicionou os valores dos índices: LVd $<$ LVAd2 < LVdf < LVAd1 (Tabela 4). Se comparados os LVAd2 e o LVdf, com teor inicial semelhante, a diferença no índice responde à presença de vermiculita com hidróxido-entre-camadas no primeiro (Amaral, 2000); este mineral incrementa a capacidade de reposição de potássio 
Tabela 5. Contrastes médios comparando-se a relação entre o maior teor e o teor médio de amônio, nitrato, potássio e fósforo, aplicados como pulso único, considerando-se os solos que apresentaram homogeneidade de variância

\begin{tabular}{|c|c|c|}
\hline Nutriente & Contraste & Valor \\
\hline \multirow{3}{*}{$\mathrm{N}-\mathrm{NH}_{4}^{+}$} & -3 LVAd1 + LVAd2 + LVdf + LVd & $0,08 * *$ \\
\hline & -2 LVAd2 + LVdf + LVd & $0,07 * *$ \\
\hline & $-L V d f+L V d$ & $0,07 * *$ \\
\hline \multirow{3}{*}{$\mathrm{N}-\mathrm{NO}_{3}^{-}$} & -3 LVAd1 + LVAd2 + LVdf + LVd & $0,03^{*}$ \\
\hline & -2 LVAd2 + LVdf + LVd & $-0,18^{* *}$ \\
\hline & $-L V d f+L V d$ & $0,22 * *$ \\
\hline \multirow{3}{*}{ K } & -3 LVAd1 + LVAd2 + LVdf + LVd & $-0,76^{* *}$ \\
\hline & -2 LVAd2 + LVdf + LVd & $-0,20 * *$ \\
\hline & $-L V d f+L V d$ & $-0,50 * *$ \\
\hline \multirow{2}{*}{ P } & -LVAd1 + LVd & $-3,19 * *$ \\
\hline & - LVAd2 + LVdf & $-0,57 * *$ \\
\hline
\end{tabular}

diminuindo, assim, a desuniformidade na distribuição do nutriente nas colunas de solos.

A presença de ilita no LVd também contribui para o menor valor do índice apresentado por este solo; neste sentido, alguns autores têm mostrado a capacidade de reposição de potássio pela ilita presente no solo (Araújo et al., 2003; Corrêa et al., 2003), aumentando os teores de potássio.

$\mathrm{O}$ fósforo, devido à sua localização no anel superficial e sem movimento nas colunas em razão da baixa quantidade de água acrescentada, apresenta índices que somente são reflexo da capacidade de fixação do nutriente pelos solos em estudo (Tabelas 4 e 5). O fato do LVd apresentar menor índice que o LVdf deve ser justificado pela maior disponibilidade inicial do primeiro. Pode-se indicar, então, que o índice proposto é de importância para estimar a desuniformidade de distribuição de solutos, podendo ser aplicado a íons móveis (amônio e nitrato) ou medianamente móveis (potássio), casos em que deve se considerar a interação do íon com os constituintes da fase sólida e seu teor inicial no solo; este índice não apresenta significado prático no estudo de íons muito pouco móveis no solo como, por exemplo, o fósforo; a extensão na interpretação do índice para esses últimos exigiria a incorporação de volumes expressivos de água ou solução.

Tem-se, na Tabela 6, os contrastes que comparam o índice nos tratamentos em que os nutrientes foram aplicados como pulso único $\left(\mathrm{F}_{2} \mathrm{D}, \mathrm{F}_{3} \mathrm{D}, \mathrm{F}_{4} \mathrm{D}, \mathrm{F}_{2} \mathrm{D}_{1 / 2} \mathrm{~F}_{3} \mathrm{D}_{1 / 2}, \mathrm{~F}_{3} \mathrm{D}_{1 / 2} \mathrm{~F}_{4} \mathrm{D}_{1 / 2}\right.$ e $\left.\mathrm{F}_{2} \mathrm{D}_{1 / 3} \mathrm{~F}_{3} \mathrm{D}_{1 / 3} \mathrm{~F}_{4} \mathrm{D}_{1 / 3}\right)$; nessas condições, a localização em maior profundidade foi obtida com a maior concentração no pulso e com a maior lâmina de água posterior à sua aplicação. Nota-se que os tratamentos que permitem que os nutrientes se desloquem em maior profundidade, deveriam apresentar menor valor do índice e, em consequência, maior uniformidade na sua distribuição; esta observação é confirmada para o amônio e o nitrato, quando comparada com a concentração no pulso (Tabela 6); no que diz respeito à lâmina de água posterior à aplicação do pulso, os resultados, quando estatisticamente significativos, ratificam as observações feitas previamente, enquanto a comprovação plena se verifica com o potássio, nutriente de mobilidade intermedi-
Tabela 6. Contrastes médios comparando-se a relação entre o maior teor e 0 teor médio de amônio, nitrato, potássio e fósforo, aplicados como pulso único e se considerando a fração da lâmina de irrigação (F) em que o nutriente foi aplicado em dose integral (D), ou fracionada em duas $\left(D_{1 / 2}\right)$ ou em três vezes $\left(D_{1 / 3}\right)$

\begin{tabular}{|c|c|c|c|c|c|}
\hline Tratamento & $C_{1}$ & $\mathrm{C}_{2}$ & $C_{3}$ & $\mathrm{C}_{4}$ & $C_{5}$ \\
\hline $\mathrm{F}_{2} \mathrm{D}$ & -1 & 0 & -2 & 0 & 0 \\
\hline $\mathrm{F}_{3} \mathrm{D}$ & -1 & 0 & 1 & -1 & 0 \\
\hline $\mathrm{F}_{4} \mathrm{D}$ & -1 & 0 & 1 & 1 & 0 \\
\hline $\mathrm{F}_{2} \mathrm{D}_{1 / 2} \mathrm{~F}_{3} \mathrm{D}_{1 / 2}$ & 1 & -1 & 0 & 0 & -1 \\
\hline $\mathrm{F}_{3} \mathrm{D}_{1 / 2} \mathrm{~F}_{4} \mathrm{D}_{1 / 2}$ & 1 & -1 & 0 & 0 & 1 \\
\hline \multirow{2}{*}{$\mathrm{F}_{2} \mathrm{D}_{1 / 3} \mathrm{~F}_{3} \mathrm{D}_{1 / 3} \mathrm{~F}_{4} \mathrm{D}_{1 / 3}$} & 1 & 2 & 0 & 0 & 0 \\
\hline & & $\mathrm{N}-\mathrm{NH}_{4}^{+}$ & & & \\
\hline LVAd1 & $0,14 * *$ & $0,13^{* *}$ & $0,07 *$ & 0,06 & 0,05 \\
\hline LVAd2 & $0,19 * *$ & $0,08 *$ & 0,04 & $0,15^{* *}$ & 0,03 \\
\hline LVdf & $0,13 * *$ & $0,17 * *$ & 0,06 & 0,02 & 0,04 \\
\hline \multirow[t]{2}{*}{$\underline{\mathrm{LVd}}$} & $0,12 * *$ & $0,15^{* *}$ & $-0,05$ & 0,02 & 0,05 \\
\hline & & $\mathrm{N}-\mathrm{NO}_{3}^{-}$ & & & \\
\hline LVAd1 & $0,32 * *$ & $0,23 * *$ & $-0,02$ & 0,07 & $0,13^{* *}$ \\
\hline LVAd2 & $0,14 * *$ & 0,06 & $0,17^{* *}$ & $-0,01$ & 0,07 \\
\hline LVdf & $0,13 * *$ & $0,18 * *$ & 0,01 & $0,11 * *$ & $0,22 * *$ \\
\hline \multirow[t]{2}{*}{$\underline{\mathrm{LVd}}$} & $0,25 * *$ & $0,22 * *$ & $-0,08 *$ & $-0,14 * *$ & $0,30 * *$ \\
\hline & & $\mathrm{K}$ & & & \\
\hline LVAd1 & $0,91 * *$ & $0,35 * *$ & $0,95 * *$ & $0,32 * *$ & $0,30 * *$ \\
\hline LVAd2 & $0,58 * *$ & $0,36 * *$ & $0,39 * *$ & $0,22 * *$ & $0,25^{* *}$ \\
\hline LVdf & $0,50 * *$ & $0,08 * *$ & $0,43 * *$ & $0,24 * *$ & $0,10 * *$ \\
\hline \multirow[t]{2}{*}{ LVd } & $0,31 * *$ & $0,17 * *$ & $0,12 * *$ & $0,08 * *$ & $0,14 * *$ \\
\hline & & $P$ & & & \\
\hline LVAd1 & $-0,02$ & 0,00 & 0,03 & 0,04 & 0,00 \\
\hline LVAd2 & 0,07 & 0,07 & 0,01 & $-0,06$ & $-0,01$ \\
\hline LVdf & $-0,04$ & 0,07 & 0,10 & $-0,03$ & $-0,02$ \\
\hline$\underline{\mathrm{LVd}}$ & $0,05 * *$ & $0,07 * *$ & 0,03 & 0,01 & 0,05 \\
\hline
\end{tabular}

ária neste ensaio, considerando-se que os valores analisados corroboram com essa suposição, para todos os tratamentos aplicados. A utilização deste índice para fósforo não é apropriada nas condições experimentais, pela sua reduzida mobilidade, como previamente analisado.

\section{CONCLUSÕES}

1. Recomenda-se, para avaliação da movimentação de nitrato, amônio e potássio em ensaio em colunas de solo, a utilização da relação teor médio/maior teor como índice de uniformidade de distribuição de nutrientes.

2. O índice de uniformidade de distribuição dos nutrientes (maior teor/teor médio) varia com o momento de aplicação dos nutrientes e o fracionamento da dose.

3. O coeficiente de variação mostrou-se menos adequado frente à relação teor médio/maior teor, para mostrar a uniformidade de distribuição de nutrientes em resposta aos tratamentos.

4. A interpretação do índice de uniformidade de distribuição de nutrientes ao longo de colunas, deve levar necessariamente em consideração o tipo de nutriente, a sua interação com a fase sólida e o seu teor inicial no solo.

5. A utilização do índice para fósforo nas condições ex- 
perimentais não é recomendada; a sua utilização estaria condicionada à aplicação de volumes expressivos de água.

\section{AGRADECIMENTOS}

À Fundação de Amparo à Pesquisa de Minas Gerais FAPEMIG, pela concessão da bolsa durante o curso de Doutorado

\section{LITERATURA CITADA}

Alvarez V., V. H.; Alvarez, G. A. M. Comparação de médias por teste de hipóteses? Contrastes! Boletim Informativo, Sociedade Brasileira de Ciência do Solo, V.31, n.1, p.24-34, 2006.

Alvarez V., V. H.; Novais, R. F.; Dias, L. E.; Oliveira, J. A. Determinação e uso do fósforo remanescente. Boletim Informativo, Sociedade Brasileira de Ciência do Solo, v.25, n.1, p.27-33, 2000

Amaral, G. Características químicas e físicas de diferentes classes de solos da zona metalúrgica mineira e produtividade de eucalipto. Viçosa: UFV, 2000. 89p. Dissertação Mestrado

Araújo, C. A. S. Movimento de fósforo e de macronutrientes catiônicos em agregados de um Latossolo Vermelho-Escuro. Viçosa: UFV, 1997. 124p. Dissertação Doutorado

Araújo, C. A. S.; Ruiz, H. A.; Silva, D. J.; Ferreira, P. A.; Alvarez, V. V. H.; Bahia Filho, A. F. C. Eluição de magnésio, cálcio e potássio de acordo com o tempo de difusão em colunas com agregados de um Latossolo Vermelho distrófico típico. Revista Brasileira de Ciência do Solo, v.27, n.2, p.231-238, 2003.

Braga, J. M.; Defelipo, B. V. Determinação epectrofotométrica de fósforo em extratos de solo e material vegetal. Revista Ceres, v.21, n.113, p.73-85, 1974.

Carrijo, O. A.; Silva, W. L. C.; Marquelli, W. A.; Silva, H. R. Tendências e desafios da fertigação no Brasil. In: Folegatti, M. V. (ed) Fertigação: Citrus, flores, e hortaliças. Guaíba: Livraria e Editora Agropecuária, 1999. p.155-169.

Correa, M. M.; Ker, J. C.; Ruiz, H. A. ; Mendonça, E. S. Atributos físicos, químicos e mineralógicos dos solos da região das Várzeas de Sousa-PB. Revista Brasileira de Ciência do Solo, SBCS, v. 27, n. 2, p. 311-324, 2003.Costa, S. N.; Martinez, M. A.; Matos, A. T.; Ramos, V. B. N. Mobilidade de nitrato em colunas de solo sob condições de escomaneto não permanente. Revista Brasileira de Engenharia Agrícola e Ambiental, v.3, n.2, p.190-194, 1999.

Costa, S. N.; Marlins, M. A.; Matos, A. T.; Ramos, V. B. N. Mobilidade de nitrato em colunas de solo sob condições de escoamento não permanente. Revista Brasileira de Engenharia Agrícola e Ambiental, v.3, n.2, p.190-194, 1999.

Defelipo, B. V.; Ribeiro, A. C. Análise química do solo. Viçosa: UFV, 2.ed., 1997. 26p. Boletim de Extensão, 29.

Donagemma, G. K. Pré-tratamento na análise textural visando a minimização do pseudo-silte em Latossolos de Minas Gerais. Viçosa: UFV, 2000. 89p. Dissertação Mestrado
EMBRAPA - Empresa Brasileira de Pesquisa Agropecuária. Manual de métodos de análise de solo. 2.ed. Rio de Janeiro: Empresa Brasileira de Pesquisa Agropecuaria 1997. 212p.

Fesch, C.; Lehman, P.; Haderlein, S. B.; Hinz, C.; Scharzenbach, R. P.; Fluhler, H. Effect of water content on solute transport in a porous medium containing reactive micro-aggregates. Journal of Contaminant Hydrology, v.33, n.1, p.211-230, 1998.

Frizzone, J. A.; Zanini, J. R.; Paes, L. A. D.; Nascimento, V. M. Fertigação mineral. Ilha Solteira: UNESP, 1985. 31p.

Grant, R. F.; Heaney, D. J. Inorganic transformation and transport in soils: mathematical modeling in ecosystem. Soil Science. Society of American Journal, v.61, n.3, p.752-764, 1997.

Hernandez, F. B. T. Potencialidades da fertigação. In: Vitti, G. C.; Boareto, A. E. (ed.) Fertilizantes fluidos. Piracicaba: ESALQ/ USP, 1993. p.199-210.

Jacobsen, O. H.; Moldrup, P.; Larsen, C.; Konnerup, L.; Petersen, L. W. Particle transport in macro porous of undisturbed soil columns. Journal of Hydrology, v.196, n.1, p.185-203, 1997.

Jensen, M. B.; Jorgesen, P. R.; Hansen, H. C. B.; Nielsen, N. E. Bio pore mediated subsurface transport of dissolved orthophosphate. Journal Environmental Quality, v.27, n.5, p.1130-1137, 1998.

Kempers, A. J.; Zewers, W. L. Ammonium determination in soil extracts by salicylate method. Communication Soil Science and Plant Analysis, v.17, n.7, p.715-723, 1986.

Kirkham, D.; Powers, W. L. Advanced soil physics. New York: John Wiley-Interscience, 1972. 534p.

Kirkby, C. A.; Smythe, L. J.; Cox, J. W.; Chittleborough, D. J. Phosphorus movement down top sequence from a landscape with texture contrast soils. Australian Journal of Soil Research, v.35, n.2, p.399-417, 1997.

Oliveira, E. M. M.; Ruiz, H. A.; Ferreira, P. A.; Alvarez, V. H.; Borges Júnior, J. C. F. Fatores de retardamento e coeficientes de dispersão-difusão de fosfato, potássio e amônio em solos de Minas Gerais. Engenharia Agrícola e Ambiental, v.8, n.2, p.196-203, 2004

Padilla, I. Y.; Jim Yeh, T. C.; Conklin, M. H. The effect of water content on solute transport in unsatured porous media. Water. Resource Research. v.35, p.3303-3313, 1999.

Pinheiro, R. B. Mobilidade de nitrato em resposta a propriedades eletroquímicas de solos com carga variável. Viçosa: UFV, 2002. 76p. Dissertação Mestrado

Pinto, J. M. Fertigação em fruticultura irrigada. Item, V.49, p.14-23, 2001.

Qafoku, N. P.; Sumner, M. E. Retention and transport of calcium nitrate in variable charge sub soils. Soil Science, v.166, n.5, p.297-307, 2001.

Qafoku, N. P.; Sumner, M. E.; Radcliffe, D.E. Anion transport in columns of variable charge sub soils: nitrate and chloride. Journal Environmental Quality, v.29, n.2, p.484-493, 2000.

Ruiz, H. A. Incremento da exatidão da análise granulométrica do solo por meio da coleta da suspensão (silte + argila). Revista Brasileira de Ciência do Solo, v.29, n.2, p.297-300, 2005.

Santos Neto, J. A. Taxas de recuperação de zinco, cobre e boro por diferentes extratores em solos da Bahia e de Minas Gerais. Viçosa: UFV, 2003. 50p. Dissertação Mestrado

Schumman, L. M. Phosphate and nitrate movement trough simulated golf greens. Water Air Soil Pollution, v.129, p.305-318, 2001. 
Shiptalo, M. J.; Dick, W. A.; Edwards, W. M. Conservation tillage and macropore factors that affect water movement and the fate of chemicals. Soil Tillage Research, v.53, n.3, p.167-183, 2000.

Silva, A. S. Movimentação de amônio, nitrato, potássio e fósforo aplicados por fertigação em Latossolos. Viçosa: UFV, 2004. 58p. Dissertação Mestrado
Yang, J. E.; Skogley. E. O.; Schaff, B. E.; Kim, J. J. A simple spectrophotometric determination of nitrate in water, resin and soil extracts. Soil Science. Society American Journal, v.62, n.4, p.1108-1115, 1998.

Yeomans, J. C.; Bremner, J. M. A rapid and precise method for routine determination of carbon in soil. Communication Soil Science Plant Analysis, v.19, n.13, p.1467-1476, 1988. 